\title{
Auswertung von 3ojährigen Aufzeichnungen der Regenmeßstation Rotmonten in St. Gallen
}

\author{
Von Dipl.-Ing. A. HörLER
}

Chef der technischen Abtcilung der EAWAG

Mitteilung Nr. 132 der Eidg. Anstalt für Wasserversorgung, Abwasserreinigung und Gewässerschutz an der E'TH, Zürich. Direktor: Prof. Dr. O.JAAG

Manuskript cingegangen am 26. September 1957 


\section{Einleitung}

Es sind jetzt mehr als I2 Jahre her, seit Dipl.-Ing. A. Kropf [I] ${ }^{1}$, damaliger Chef der Beratungsstelle für Abwasserreinigung und Trinkwasserversorgung an der ETH, in der Schweizerischen Bauzeitung seine vielbeachteten Auswertungsresultate der Regenstreifen der Stadt Zürich veröffentlichte. Diese dienten zur hydraulischen Berechnung von Kanalisationen und wurden seither nicht nur für die Region 'Zürich angewendet, sondern mangels systematischer Auswertung von SchreibregenmesserAufzeichnungen anderer Städte auch bei Kanalisationsprojekten von Gemeinden anderer Kantone zugrunde gelegt.

Neben diesen Auswertungen dienen in verschiedenen Städten der Schweiz Regenintensitätskurven der Dimensionierung von Kanalisationen, die nach ganz verschiedenen Grundsätzen und Richtlinien ausgewertet worden sind, so dass ein Vergleich mit der systematischen Auswertung der Regenintensitäten der Stadt Zürich nicht möglich ist. Da zudem oft Auswertungsmethoden angewendet wurden, die bezüglich der Häufigkeit des Eintretens von Starkregenfällen bestimmter Intensität und Dauer ein ungenaues oder sogar falsches Bild ergeben, ist bei der Verwendung solcher Intensitätskurven Vorsicht am Platze.

Während in Deutschland eine einheitliche Auswertung der Niederschläge von 144 Regenmeßstellen vorliegt [2], die nach dem von Prof. REINHOLD, Darmstadt, veröffentlichten Auswerteverfahren [3] vorgenom-

1) Die Ziffern in eckigen Klammern verweisen auf das Literaturverzeichnis, Seite 40. 
men wurde, so dass die Ergebnisse untereinander verglichen werden können, besteht für die Schweiz keine ähnliche Auswertung, mit Ausnahme der für Zürich erhobenen Werte. Es wäre deshalb sehr zu begrüssen, wenn die Ermittlung der Regenintensitäten nach dem von REINHOLD vorgeschlagenen Verfahren, das sich speziell für wasserwirtschaftliche Zwecke eignet, auch für andere Ortschaften vorgenommen würde, die über einen seit mindestens 30 Jahren in Tätigkeit befindlichen Schreibregenmesser (Pluviographen) verfügen.

Als Beitrag zu dieser umfassenden Auswertearbeit seien im folgenden die Auswertungsresultate der Regenbeobachtungen von St. Gallen bekanntgegeben, die ebenfalls nach den Anweisungen zur Auswertung von Scbreibregenmesser-Aufzeichnungen vorgenommen wurden.

\section{Allgemeine Grundlagen}

Da heute die Dimensionierung von Kanalisationen nach der Fliesszeitmethode vorherrscht, ist die Kenntnis des Zusammenhanges zwischen Regenintensität und Regendauer für die der Berechnung zugrunde gelegte jährliche Regenhäufigkeit von wesentlicher Bedeutung.

Die Regenintensität wird angegeben entweder als Regenstärke $i$ in Millimeter pro Minute, oder als Regenspende $r$ in Liter pro Sekunde je Hektare. Die letzterwähnte Angabe ist die häufigere, da sie sich zur direkten Berechnung der Regenwassermenge an einer bestimmten Stelle eines Kanalisationsnetzes am besten eignet. In der Schweiz ist es gebräuchlich, die Regenspende ebenfalls mit dem übergeordneten Begriff als «Regenintensität» zu bezeichnen.

Die Regenstärke ergibt sich aus der Beziehung

$$
i=\frac{N}{T}[\mathrm{~mm} / \mathrm{min}] \text {, }
$$

die Regenspende aus der Beziehung

$$
r=166,7 \frac{N}{T}[1 / \mathrm{s} \cdot \mathrm{ha}],
$$

wobei $N=$ Regenhöhe in Millirneter und $T=$ Regendauer in Minuten.

Die jährliche Häufigkeit $n$ gibt an, wie oft innert eines Jahres ein Regen erreicht oder überschritten wird. Umgekehrt gibt die Zahl $z$ an, innert wieviel Jahren ein Regen bestimmter Intensität und Dauer erreicht wird. Es gilt somit die Beziehung

$$
n z=1 \text {. }
$$


Jede bestimmte Regenspende $r$ ist durch zwei Indizes charakterisiert, nämlich durch die 'Zahl $z$ und $T$, das heisst durch die Anzahl Jahre, innert welchen durchschnittlich das Ereignis einmal eintritt, und durch die Regendauer in Minuten. Diese Indizes werden wie folgt angeordnet: $r_{T}^{(z)}$. So bedeutet zum Beispiel $r_{15}^{(5)}$ denjenigen Regen von Is Minuten Dauer, der alle 5 Jahre einmal erreicht wird.

Für das eingehendere Studium sämtlicher Fragen bezüglich der Auswerteverfahren, Genauigkeit, Fehlerausgleichung usw. sei auf die Fachliteratur verwiesen $[\mathrm{I}-S]$.

\section{Das zur Auswertung zur Verfügung stehende Material}

Die Stadt St.Gallen besitzt Schreibregenmesser in Rotmonten und Sankt Georgen. Da die Aufzeichnungen in Rotmonten seit I924 bis I953 fast durchgehend vorhanden waren, wurde die Meßstelle Rotmonten zur Auswertung herangezogen. Nur die Regenfälle in den Jahren 1933 und 1934 mussten von der Meßstelle St.Georgen übernommen werden, da die Regenstreifen Rotmonten in diesen Jahren merkbare Lïcken aufwiesen. Zudem mussten zur Deckung lückenhafter Monate noch auf Regenstreifen der Monate Juni (St.Georgen) und August (Rotmonten) des Jahres 1954 gegriffen werden. Es zeigt sich immer wieder, dass es schwierig ist, lückenlose Aufzeichnungen der Regenfälle über 30 Jahre zu erhalten. Obgleich auch in St.Gallen Regen nicht über das ganze Jahr registriert werden, sondern nur während der Sommermonate (Mai bis Oktober/November), genügte das vorhandene Auswertematerial, da die für die Auswertung massgebenden Starkregen auf die Sommermonate entfallen.

\section{Die Auswertung}

Diese erfolgte nach dem von REINHOLD entwickelten Verfahren. Aus Mangel an Arbeitskräften musste jedoch danach getrachtet werden, mit einem Minimum an Auswertearbeit auszukommen. Deshalb wurden die untersuchten Starkregen nur für I0, 20, 30 und 60 Minuten Regendauer ausgewertet. Diese bewusste Reduktion der Auswertearbeit geht selbstverständlich auf Kosten der Verfeinerung der Intensitätskurve. Die hydraulische Berechnung der Kanalisationen weist jedoch Faktoren auf, die bedeutend unsicherer sind als die Regenintensität, selbst bei einer Reduktion der Auswertearbeit auf 4 Punkte pro Intensitätskurve, weshalb die 
Genauigkeit vorliegender Auswertung für die praktischen Zwecke genügen dürfte.

\section{Resultate der Auswertung}

Die Regenspenden $r$, geordnet nach Regendauer $T$ und der jährlichen Häufigkeit $n$ bzw. der Anzahl Jahre z, innert welchen eine Überschreitung $z u$ erwarten ist, gehen aus der Tabelle I hervor. In dieser Tabelle sind die ausgewerteten Originalwerte ohne jede Ausgleichung der Resultate wiedergegeben.

Tabelle 1 .

\begin{tabular}{|c|c|c|c|c|c|c|c|c|c|}
\hline $\begin{array}{c}n \\
\text { bzw. } \\
z\end{array}$ & $\begin{array}{c}2 \\
1 / 2\end{array}$ & $\begin{array}{l}1 \\
1\end{array}$ & $\begin{array}{c}1 / 2 \\
2\end{array}$ & $\begin{array}{c}1 / 3 \\
3\end{array}$ & $\begin{array}{c}1 / 5 \\
5\end{array}$ & $\begin{array}{c}1 / 6 \\
6\end{array}$ & $\begin{array}{c}1 / 10 \\
10\end{array}$ & $\begin{array}{c}1 / 15 \\
15\end{array}$ & $\begin{array}{c}1 / 30 \\
30\end{array}$ \\
\hline Regen- & \multicolumn{9}{|c|}{ Regenspende $[1 / \mathrm{s} \cdot \mathrm{ha}]$} \\
\hline $\begin{array}{c}T \\
\min \end{array}$ & $r_{T}^{(1 / 2)}$ & $r_{T}^{(1)}$ & $r_{T}^{(2)}$ & $r_{T}^{(3)}$ & $r_{T}^{(5)}$ & $r_{T}^{(\boldsymbol{6})}$ & $r_{T}^{(10)}$ & $\gamma_{T}^{(15)}$ & $r_{T}^{(30)}$ \\
\hline 10 & 123 & 170 & 217 & 250 & 284 & 333 & 374 & 377 & 384 \\
\hline 20 & 87 & 118 & 158 & 197 & 217 & 225 & 23 & 250 & 250 \\
\hline 30 & 63,5 & 83,3 & 130 & 150 & 160 & 178 & 183 & 195 & 214 \\
\hline 60 & 40,0 & 51,7 & 73,5 & 81,7 & 91,7 & 105 & 112 & 112 & 114 \\
\hline
\end{tabular}

Die Ausgleichung der erhaltenen Werte erfolgte derart, dass vorerst die je 9 Werte für $T=$ Io, 20, 30 und 60 Minuten als Funktion von $z$ dargestellt wurden (Abb.I). Dabei zeigte sich, dass eine Gleichung

$$
r=\frac{z}{a+b z},
$$

wobei $a$ und $b$ für einen Regen bestimmter Dauer Konstanten bedeuten, geringere Abweichungen aufweist als die Darstellung im logarithmischen Netz.

Um einen Ausgleich nach der Korrelationsrechnung zu ermöglichen, wurden die Auswerteergebnisse in einem Koordinatennetz mit $z / r$ als Ordinate und $z$ als Abszisse dargestellt. Damit ergeben sich für die Werte bestimmter Regendauer gerade Linien, da

$$
y=\frac{z}{r}=a+b z
$$

wird. In Abbildung 2 sind die Beobachtungswerte samt den Ausgleichsgeraden dargestellt. Jede Gerade ist somit durch 9 Punkte festgelegt, an- 


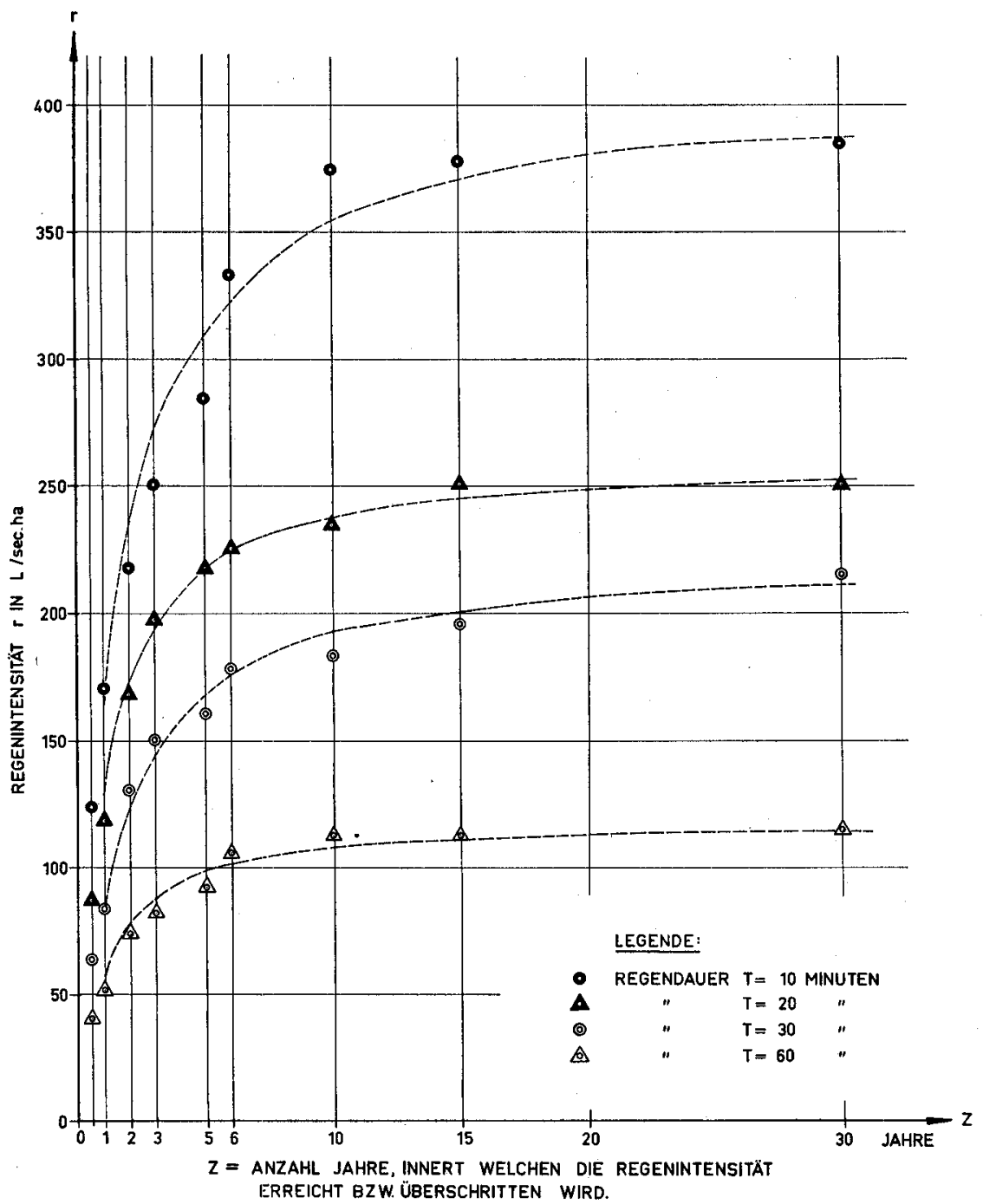

Abbildung 1

Regenintensitätskurven $r=f(z)$.

Die gestrichelten Kurven entsprechen den in Abbildung 2 dargestellten ausgeglichenen Geraden. 
$\mathrm{XX}, 1958$

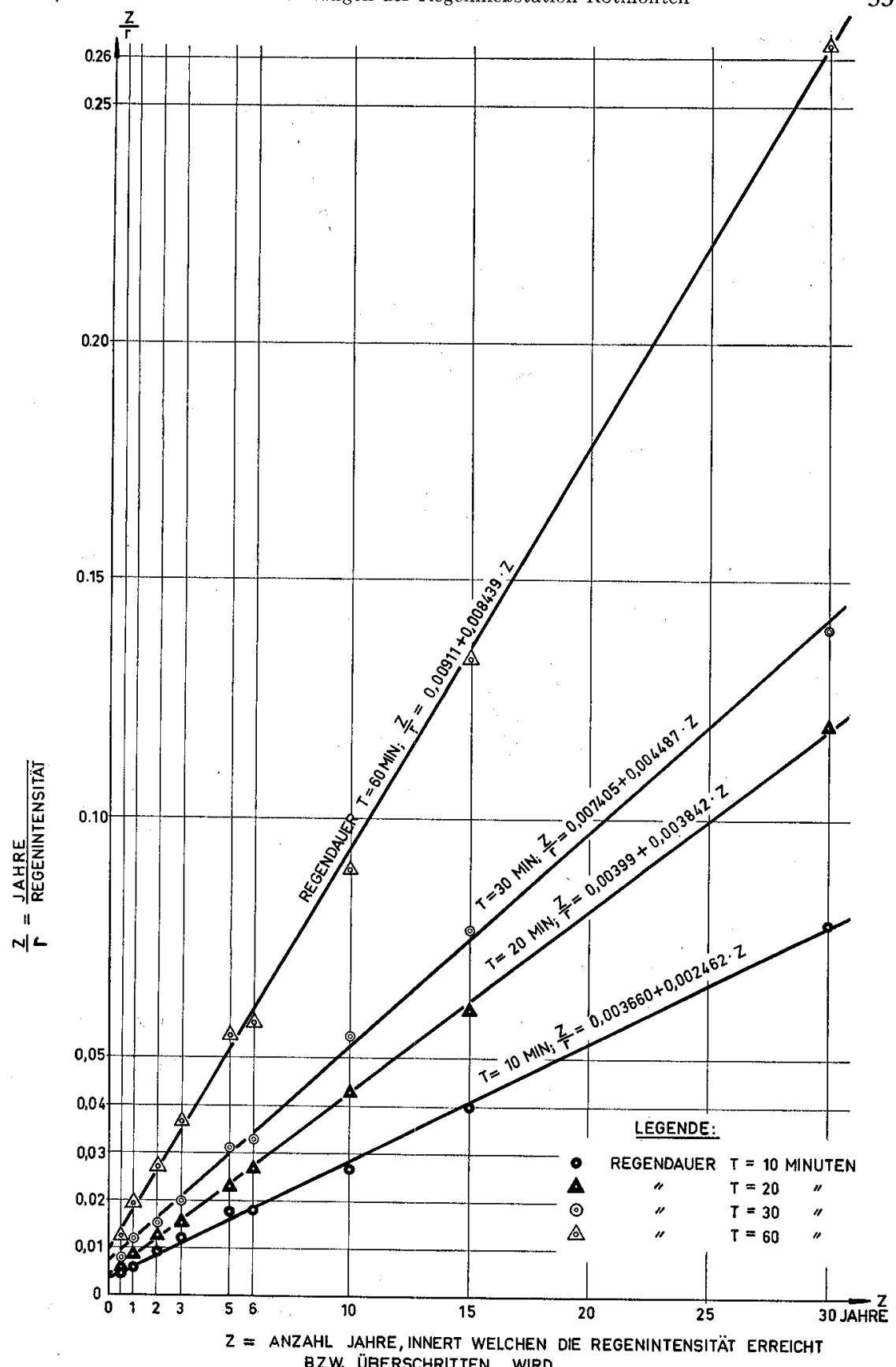

Abbildung 2

Regenintensitätskurven $z / r=f(z)$. 


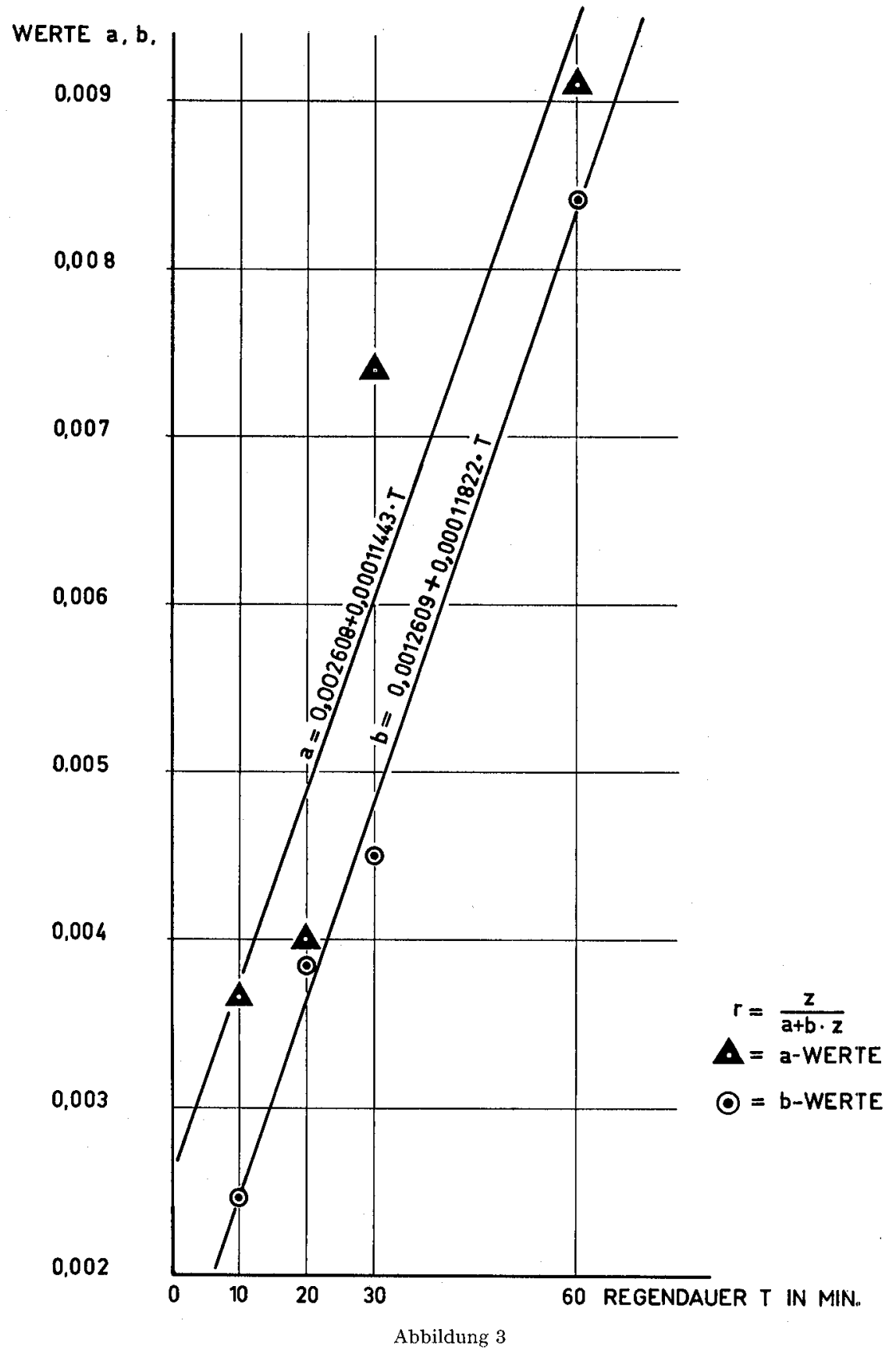

Werte $a$ und $b$ in Funktion der Regendauer $T$. 
statt nur durch 4 Punkte, wie dies bei Wahl von $z$ als Parameter der Fall gewesen wäre.

Um die in der Kanalisationstechnik gebräuchliche Darstellung (Ordinate $=$ Regenintensität $r$, Abszisse $=$ Regendauer $T$ in Minuten) und zugleich eine Schar ausgeglichener Kurven $r=$ Funktion von $T$ mit $z$ als Parameter zu erhalten, wurde vorerst die den Regenzeiten 10, 20, 30 und 60 Minuten zugeordneten Konstanten $a$ und $b$ in Funktion von $T$ dargestellt und für $a$ und $b$ ein ausgeglichenes Geradenpaar erhalten (Abb. 3 ), das die Gleichungen

$$
\begin{aligned}
& a=\alpha_{1}+\alpha_{2} T=0,002608+0,00011443 T, \\
& b=\beta_{1}+\beta_{2} T=0,0012609+0,00011822 T
\end{aligned}
$$

befolgt, wobei $\alpha_{1}, \alpha_{2}, \beta_{1}, \beta_{2}$ wieder konstante Werte bedeuten.

Setzt man die Gleichungen ( $2 \mathrm{a}$ ) und ( $2 \mathrm{~b}$ ) in Gleichung ( $\mathrm{I}$ ) ein, so erhalten wir

$$
\gamma=\frac{z}{\left(\alpha_{1}+\beta_{1} z\right)+\left(\alpha_{2}+\beta_{2} z\right) T}
$$

oder für einen bestimmten Wert von $z(z=$ const $)$ :

$$
r_{T}=\frac{C}{B+T} \quad(C, B=\text { const })
$$

entsprechend der von REINHOLD [3] ermittelten Beziehung, die sich für die Regenintensitäten von Deutschland als Spezialfälle der allgemeinen Gleichung ( 5 )

$$
r=\frac{C}{(B+T)^{\alpha}}
$$

ergeben. Durch die angegebenen Zahlenwerte der Gleichungen (2a) und (2b) kann die ausgeglichene Regenintensität $r$ für jede beliebige Regendauer und jede beliebige Anzahl Jahre z, in denen der gewählte Regen erreicht wird, errechnet werden.

In Abbildung 4 sind die ermittelten Werte und die ausgeglichenen Kurven $r$ als Funktion der Regendauer $T$ dargestellt. Die nachfolgende Tabelle 2 gibt für bestimmte Werte von $T$ und $z$ die zugehörigen ausgeglichenen Werte für die Regenintensitäten wieder. 


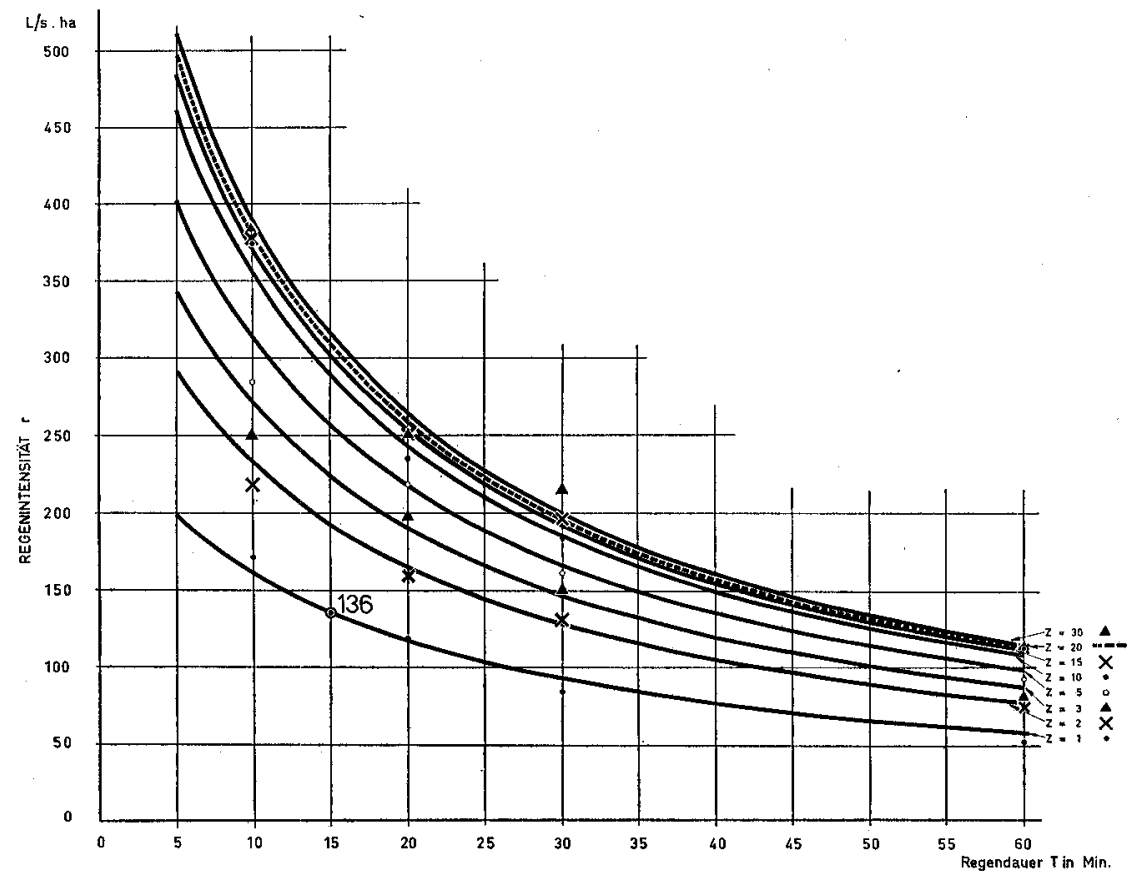

Abbildung 4

Regenintensitätskurven $r$ in Funktion der Regendauer $T$.

Tabelle 2

\begin{tabular}{|c|c|c|c|c|c|c|c|c|}
\hline $\begin{array}{c}n \\
\mathrm{~b} z \mathrm{w} \\
z\end{array}$ & $\begin{array}{l}1 \\
1\end{array}$ & $\begin{array}{c}1 / 2 \\
2\end{array}$ & $\begin{array}{c}1 / 3 \\
3\end{array}$ & $\begin{array}{c}1 / 5 \\
5\end{array}$ & $\begin{array}{c}1 / 10 \\
10\end{array}$ & $\begin{array}{c}1 / 15 \\
15\end{array}$ & $\begin{array}{c}1 / 20 \\
20\end{array}$ & $\begin{array}{c}1 / 30 \\
30\end{array}$ \\
\hline $\begin{array}{c}\text { Regen- } \\
\text { dauer } \\
T \\
\text { min }\end{array}$ & \multicolumn{8}{|c|}{ Regenintensitäten $r[1 / \mathrm{s} \cdot$ ha $]$} \\
\hline 5 & 199 & 291 & 343 & 402 & 461 & 484 & 497 & 511 \\
\hline 10 & 161 & 232 & 271 & 313 & 355 & 371 & 380 & 389 \\
\hline 15 & 136 & 192 & 233 & 256 & 288 & 301 & 308 & 315 \\
\hline 20 & 117 & 165 & 190 & 217 & 243 & 253 & 258 & 264 \\
\hline 25 & 103 & 144 & 166 & 188 & 210 & 218 & 223 & 227 \\
\hline 30 & 92,2 & 128 & 147 & 166 & 185 & 192 & 196 & 200 \\
\hline 35 & 83,3 & 115 & 132 & 149 & 165 & 171 & 175 & 178 \\
\hline 40 & 75,9 & 104 & 119 & 135 & 149 & 155 & 158 & 161 \\
\hline 45 & 69,7 & 95,6 & 109 & 123 & 136 & 141 & 143 & 146 \\
\hline 50 & 64,5 & 88,2 & 101 & 113 & 125 & 129 & 132 & 134 \\
\hline 55 & 60,0 & 81,9 & 93,2 & 105 & 116 & 120 & 122 & 124 \\
\hline 60 & 56,1 & 76,4 & 86,9 & 97,6 & 108 & 111 & 113 & 115 \\
\hline
\end{tabular}


Die mittleren Abweichungen der Werte der Tabelle 2 gegenüber den Werten der Tabelle I betragen:

\begin{tabular}{|c|c|}
\hline$T[\mathrm{~min}]$ & $1 / \mathrm{s} \cdot \mathrm{ha}$ \\
\hline 10 & $\pm 19,8$ \\
20 & $\pm 8,6$ \\
30 & $\pm 8,1$ \\
\multicolumn{2}{|c|}{ bzw. insgesamt $\pm 11,7$} \\
\hline
\end{tabular}

\section{Vergleich der Regenintensitäten von Zürich mit denjenigen von St. Gallen}

Die Regenintensitäten von Zürich und St.Gallen, sowohl für die sogenannte Grundzahl $r_{15}^{(1)}$ als auch für die übrigen Häufigkeiten der Regen von Is Minuten Dauer, sind in Tabelle 3 zusammengestellt.

Tabelle 3

\begin{tabular}{|c|c|c|c|c|c|}
\hline$Z$ & $\begin{array}{c}1 \\
\text { Jahr }\end{array}$ & $\begin{array}{c}2 \\
\text { Jahre }\end{array}$ & $\begin{array}{c}5 \\
\text { Jahre }\end{array}$ & $\begin{array}{c}10 \\
\text { Jahre }\end{array}$ & $\begin{array}{c}30 \\
\text { Jahre }\end{array}$ \\
\hline & \multicolumn{5}{|c|}{ Regenintensitäten: $[1 / \mathrm{s} \cdot \mathrm{ha}] T=15 \mathrm{~min}$} \\
\hline St.Gallen & 136 & 192 & 256 & 288 & 315 \\
\hline Zürich & 107 & 135 & 161 & 192 & 230 \\
\hline \multicolumn{6}{|c|}{$\begin{array}{c}\text { St. Gallen weist somit gegenüber Zürich höhere Regenintensitäten auf, } \\
\text { und zwar um }\end{array}$} \\
\hline & $27 \%$ & $42 \%$ & $59 \%$ & $50 \%$ & $37 \%$ \\
\hline
\end{tabular}

Die Regenintensitäten gleicher Häufigkeit und gleicher Regendauer liegen also in St.Gallen zum Teil wesentlich höher als in Zürich, wie aus dem in obiger Tabelle aufgeführten Beispiel für Regen von Is Minuten Dauer hervorgeht.

Bei der statistischen Auswertung des Zahlenmaterials hat mir Herr Dr. A. KaELIN (Versuchsanstalt für Wasserbau an der ETH, Abteilung für Hydrologie) wertvolle Ratschläge erteilt. Zu danken habe ich ferner dem Tief bauamt der Stadt St.Gallen für die Úberlassung der Regen- 
streifen sowie meiner Frau und meinem Sohne für ihre Mitarbeit bei der Auswertung der Regendiagramme.

\section{LITER ATUR VERZEICHNIS}

[I] A. KROPF, Auswertung von 3ojäbrigen Aufzeichnungen der Regenmeßstation der meteorologischen Zentralanstalt Zürich, Schweiz. Bauztg. 123 (I944).

[2] F. REINHOLD, Regenspenden in Deutscbland (Grundwerte für die Entwässerungstechnik), Arch. Wasserwirtschaft, Nr. 56 (Berlin 1940).

[3] Abwasserfachgruppe der Deutschen Gesellschaft für Bauwesen e.V., Anweisung zur Auswertung pon Scbreibregenmesser-Aufzeicbnungen für wasserwirtschaftliche Zwecke (AAR 1936), Gesundheitsingenieur 60, H.2, 22/26; H.3, 40/45; H.4, 55/6I (I937).

[4] G. M. FAIR und J. C. GeYer, Water Supply and Waste-Water Disposal, (Verlag J. Wiley \& Sons, New York I954).

[5] Ven TE CHOw, Frequency Analysis of Hydrologic Data mith Special Application to Rainfall Intensities, Univ. Ill. Bull. 1953 (Juli). 